\title{
CAPACIDADES DEL EMPRENDIMIENTO EN INSTITUCIONES EDUCATIVAS DEL DISTRITO DE RIOHACHA, LA GUAJIRA COLOMBIANA
}

\section{ENTREPRENEURSHIPCAPABILITIES IN EDUCATIONAL INSTITUTIONS OF THE DISTRICT OF RIOHACHA, LA GUAJIRACOLOMBIANA}

\section{Andrés Salcedo Bruzon ${ }^{1}$}

\section{Raúl Martelo Gómez²}

\section{Delvis Muñoz Rojas ${ }^{3}$}

Universidad de La Guajira

Universidad de Cartagena

$1 \quad$ Doctor en Ciencias (Mención: Gerencia), Magister en Ingeniería de Control y Automatización de Procesos, Ingeniero Electrónico y en Telecomunicaciones. Universidad de La Guajira. Riohacha, Colombia. asalcedo@uniguajira. edu.co; https://orcid.org/0000-0002-2931-8085

2 Magister en Informática, Especialista en Telecomunicaciones, Ingeniero de Sistemas. Universidad de Cartagena. Cartagena de indias, Colombia.rmartelog1@unicartagena.edu.co; $h$ ttps://orcid.org/0000-0002-4951-0752

3 Doctora en Ciencias (Mención: Gerencia), Magister en Sistemas de Gestión, Magister en Neuropsicología, Magister Psicología Clínica, Especialista en Finanzas, Especialista en Salud Ocupacional, Psicóloga, Administradora de Empresas. Universidad de La Guajira. Riohacha, Colombia. dmunozr@uniguajira.edu.co; https://orcid.org/0000-00019445-9792

\section{RESUMEN}

El objetivo principal fue identificar las capacidades del emprendimiento en instituciones educativas en el Municipio Riohacha, Departamento de La Guajira en Colombia. El estudio es cuantitativo con modalidad descriptiva, el diseño es no experimental y transeccional, la población se comprende 100 sujetos de estudio. La recolección de la información, se realizó con base en la técnica de la encuesta y se seleccionó como 
instrumento un cuestionario con respuestas cerradas tipo Likert. Es necesario reforzar el diálogo, apertura, delegación y conocimiento; así como la estabilidad del sistema social, los niveles de conflictos intrapersonales e intragrupales, muy especialmente deberán impactarse todos los procesos que se manejan para la resolución de conflictos en las Instituciones Educativas.

\section{PALABRAS CLAVE}

Educación, Eficiencia, Emprendimiento, Participación, Productividad.

\section{ABSTRACT}

The main objective was to identify the capacities of entrepreneurship in educational institutions in the Municipality of Riohacha, Department of La Guajira in Colombia. The study is quantitative with descriptive modality, the design is nonexperimental and transectional, the population includes 100 study subjects. The information collection was carried out based on the survey technique and a questionnaire with closed Likert-type responses was selected as an instrument. It is necessary to reinforce dialogue, openness, delegation and knowledge; as well as the stability of the social system, the levels of intrapersonal and intragroup conflicts, especially all the processes that are handled for the resolution of conflicts in Educational Institutions should be impacted.

\section{KEYWORDS}

Education, Efficiency, Entrepreneurship, Participation, Productivity.

\section{INTRODUCCIÓN}

Las capacidades de emprendimiento contenidos en una teoría forman un conjunto de relaciones y conceptos, que son tan bien modelos formales, los cuales pueden ser verbales, gráficos, matemáticos y materiales, el concepto académico tradicional de la calidad, está supeditado al rendimiento de logros intelectuales sobresalientes en el avance del conocimiento (Martínez \& Garces, 2021; Linares \& Geizzelez, 2016).

Asimismo, se puede definir como la capacidad que tiene una organización, para satisfacer a los clientes identificado en forma correcta sus necesidades y expectativas (Cohen et al., 2020). Es decir, los índices de eficacia, sirven como orientación a la gerencia sobre el avance en las metas globales de una organización, esta debe ser entendida como la correspondencia entre los objetivos logrados por un programa, con las necesidades, expectativas y demandas sociales. Igualmente, las mediciones del desempeño de las organizaciones, se hacían solamente con indicadores financieros, no comprometía a toda la organización y tampoco la alineaban con sus objetivos estratégicos (Echeverry et al., 2018; Paz et al., 2015).

Por su parte, Casas et al. (2015), plantea que la eficiencia mide el grado en que se alcanzaron los objetivos o metas de una organización, sin tener en cuenta los recursos miembros de la comunidad educativa. Se expresan en términos cuantitativos, pero no miden la eficiencia en la utilización de los recursos. Es decir, la eficacia busca armonizar la organización con todas las condiciones. En este contexto, es la capacidad de lograr el efecto que se desea o se espera, sin que priven para ellos, los recursos o los medios miembros de la comunidad educativa haciendo uso de los indicadores de eficacia denominados medidas de correspondencia entre criterios y la ejecución.

En este sentido, la capacidad para ejecutar las operaciones administrativas de manera que satisfagan los criterios de actuación previamente establecidos. Entre algunos de los indicadores de eficacia, se tienen los índices de participación en el entramado educativo, índice 
de rotación y deserción de clientes entre otros. Por ello, una medición integral del desempeño de la organización se convierte en una necesidad inaplazable ante las nuevas condiciones (Rivera, 2019; Contreras et al., 2017). En palabras de Salguero et al. (2017) manifiestan que la eficacia, se refiere a la congruencia que existe entre lo planificado y los logros obtenidos sin cuestionar si dichos objetivos, son adecuados o no, en referencia al contexto o medio en la cual está inserta la acción pública.

Del mismo modo, se puede afirmar que normalmente la consigna de las organizaciones, es elaborar sus productos o prestar sus servicios a como dé lugar y se pierde vista cómo se logró el cumplimiento del objetivo propuesto, es decir, de qué manera se utilizan los recursos miembros de la comunidad educativa en el proceso productivo (Sandoval et al., 2020; Fong et al., 2017). De igual forma, la eficiencia o funcionalidad en términos de resultados obtenidos, al margen del costo que ello ha supuesto, aun cuando la variabilidad humana hace imprevisible el resultado de una estrategia o situación (Fong et al., 2019).

Por otra parte, para poder hacer efectivo el proceso participativo, en el que los objetivos de las instituciones educativas, desciendan en cascada a lo largo de toda la organización. Molina et al. (2020) establece que es un proceso participativo de arriba abajo que permite enfatizar, en todos los niveles, aquellos factores que sean críticos para el éxito. Debido a esto, para que sea efectivo el proceso participativo, en el que los objetivos organizacionales, descienden en cascada a lo, largo de toda organización, es preciso asignar un papel fundamental a los responsables, donde se designa las herramientas necesarias para mejorar su gestión.

El análisis del entorno está cifrado en identificar las oportunidades que se presentan para la organización y las amenazas que se vislumbran en el fututo. La planificación es el proceso de elección y selección entre cursos alternativos de acción con vistas a la asignación de escasos recursos, con el fin de obtener objetivos específicos, sobre la base de un diagnóstico preliminar que cubre todos los factores relevantes, que pueden ser identificados (Sánchez et al., 2017). Del mismo modo, especifica la relación de la organización con su ambiente en función de la misión, los objetivos, las estrategias y el plan de carteras (Cabana et al., 2013).

\section{METODOLOGÍA}

El presente estudio, se basa en la lógica de investigación cuantitativa, con modalidad descriptiva, el diseño es no experimental y transeccional, la población se comprende por 10 directivos, 60 docentes y 30 administrativos de las instituciones educativas Eusebio Septimio Mari y Livio Reginaldo Fischione ubicadas en Riohacha, capital del Departamento de la Guajira. La recolección de la información, se realizó con base en la técnica de la encuesta y se seleccionó como instrumento un cuestionario que está constituido por preguntas cerradas, con alternativas de respuesta: Siempre, Casi Siempre, A Veces, Casi Nunca, Nunca. Se aplica estadística descriptiva, utilizando el cuadro de rango, intervalo y categoría, sobre la base de la puntuación más alta - más baja de la escala de referencia a utilizar (5 - 1), los cuales se muestran en la Tabla 1. 
Tabla 1. Baremo para la interpretación del promedio

\begin{tabular}{|c|c|c|}
\hline Rango & Intervalo & Categoría \\
\hline I & $1,00 \leq X-1,80$ & Muy bajo nivel \\
\hline II & $1,80 \leq X-2,60$ & Bajo nivel \\
\hline III & $2,60 \leq X-3,40$ & Neutro \\
\hline IV & $3,40 \leq X-4,20$ & Alto nivel \\
\hline V & $4,20 \leq X-5,00$ & Muy alto nivel \\
\hline
\end{tabular}

Fuente: Elaboración propia

\section{RESULTADOS Y DISCUSIÓN}

La Tabla 2, muestra los resultados para las diferentes Capacidades para el Emprendimiento, así: Capacidad de Eficiencia, Capacidad Productividad y Capacidad de Participación. La ponderación respectiva fue de 2.16, 2.56 y 2.34 .

Tabla 2. Capacidades para el emprendimiento

\begin{tabular}{|c|c|c|c|c|c|c|c|c|c|c|c|c|c|c|c|c|c||}
\hline \multicolumn{10}{c||}{ Capacidad de eficiencia } & \multicolumn{10}{c||}{ Capacidad de productividad } & \multicolumn{3}{c||}{ Capacidad de participación } \\
48 & 48 & 27 & 27 & 17 & 17 & 0 & 0 & 15 & 15 & 45 & 45 & 48 & 48 & 27 & 27 & 17 & 17 \\
36 & 36 & 83 & 83 & 27 & 27 & 58 & 58 & 55 & 55 & 25 & 25 & 36 & 36 & 83 & 83 & 27 & 27 \\
0 & 0 & 0 & 0 & 52 & 52 & 22 & 22 & 30 & 30 & 0 & 0 & 0 & 0 & 0 & 0 & 52 & 52 \\
16 & 16 & 0 & 0 & 4 & 4 & 20 & 20 & 0 & 0 & 15 & 15 & 16 & 16 & 0 & 0 & 4 & 4 \\
0 & 0 & 0 & 0 & 0 & 0 & 0 & 0 & 0 & 0 & 15 & 15 & 0 & 0 & 0 & 0 & 0 & 0 \\
\hline
\end{tabular}

Fuente: Elaboración propia

De acuerdo con Geizzelez \& Soto (2021), plantea que la eficiencia mide el grado en que se alcanzaron los objetivos o metas de una organización, sin tener en cuenta los recursos empleados. Se expresan en términos cuantitativos, pero no miden la eficiencia en la utilización de los recursos. Es decir, la eficacia busca armonizar la organización con todas las condiciones. En este contexto, es la capacidad de lograr el efecto que se desea o se espera, sin que priven para ellos los recursos o los medios empleados haciendo uso de los indicadores de eficacia denominados medidas de correspondencia entre criterios y la ejecución.

Es de importancia señalar que es la capacidad de eficiencia es un proceso participativo de arriba abajo que permite enfatizar, en todos 
los niveles, aquellos factores que sean críticos para el éxito" (Torres \& Diaz, 2021; Vidal et al., 2018). Debido a esto, para que sea efectivo el proceso participativo, en el que los objetivos organizacionales descienden en cascada a lo, largo de toda organización, es preciso asignar un papel fundamental a los responsables por lo tanto los porcentajes de este indicador se ven reflejados de la siguiente manera: $15 \%$ (Siempre), $55 \%$ (Casi Siempre), $30 \%$ (A Veces), $0 \%$ (Casi Nunca) y (Nunca) se marcó con un porcentaje de $0 \%$

En este mismo orden de ideas, se presenta el indicador Capacidad Productividad, el cual presenta las siguientes alternativas: Los procesos dentro de la institución son sistemáticos. Los procedimientos de la organización son rápidos y Se siguen los pasos de cada una de las situaciones que deben resolverse en la institución.

Ahora bien, continuando con el análisis de la Tabla 2, se encuentran los ítems que responden al indicador: Capacidad de Participación, los cuales como se observa en la tabla su análisis dieron como resultados los porcentajes específicos para cada alternativa las cuales se presentan a continuación: La organización promociona recursos para el aprendizaje. El aprendizaje en la institución se auto administra; Se otorgan recursos a los miembros de la institución en la adquisición de nuevos conocimientos.

Es de importancia señalar que las capacidades para el emprendimiento es el proceso en que una de las partes percibe que la otra se opone o afecta negativamente sus intereses, implicando en todo ello una interdependencia e interacción, debido a que éstas conllevan una gama de interrelaciones que desde las actitudes hasta los actos de violencia más extremos. Asimismo, Velasco (2014), sostiene que esta situación se hace evidente en cargos que implican gran responsabilidad, puesto que, si se ejerce un liderazgo inadecuado, mala utilización de las habilidades, mala delegación de responsabilidades, relaciones laborales, ambivalentes, manipulación o coacción del trabajador, motivación deficiente, falta de capacitación y desarrollo del personal; no solo se estresa el gerente, sino que condiciona al resto del grupo al descontento.

En el ámbito laboral, según Vega \& Mera (2016), para que una situación sea catalogada como conflicto, deben cumplirse alguno (s) de los siguientes elementos básicos: a) Interacción entre dos o más participantes (no puede haber un conflicto en solitario); b) Presencia de conductas internas y/o externas incompatibles subjetivamente (diferencias de grupo); c) Intencionalidad de perjudicar al otro o atribución de tal intencionalidad; d) Utilización directa o indirecta del poder (sensación de abuso de poder por parte del agraviado) y e) Ineficacia normativa.

\section{CONCLUSIONES}

Es necesario reforzar el diálogo, apertura, delegación y conocimiento. Así mismo dentro de la dimensión elementos el indicador: estabilidad del sistema social. Igualmente, dentro de la dimensión: niveles de conflictos deberán reforzarse los niveles intrapersonales e intragrupales. Muy especialmente deberán ser reforzados todos los procesos que se manejan para la resolución de conflictos en las Instituciones Educativas objeto de estudio. El reforzamiento de los indicadores señalados ayudara a enfrentar o hallar soluciones para los asuntos en litigio mediante un proceso serio y eficiente que permita conseguir los arreglos necesarios a los problemas sociales y educativos que se presenten. 


\section{REFERENCIAS BIBLIOGRÁFICAS}

Cabana, R., Cortes-Castillo, Ivan, Plaza-Pasten, Diego, Castillo-Vergara, Mauricio, \& AlvarezMarin, Alejandro. (2013). Análisis de Las Capacidades Emprendedoras Potenciales y Efectivas en Alumnos de Centros de Educación Superior. Journal of Technology Management \& Innovation, 8(1), 65-75.

Casas, J., Repullo José Ramón, Lorenzo Susana Y Cañas Juan José (2015). Dimensiones y Medición de la Calidad de Vida Laboral en Profesionales Sanitarios. Revista de Administración Sanitaria, 23(2).

Cohen, J., Linares-Morales, J., \& Briceño-Ariza, L. (2020). Caracterización de la cultura innovativa en la cooperación universidadempresa. IPSA Scientia, Revista científica Multidisciplinaria, 5(1), 46-53.

Contreras, J., Wilches-Duran, Sandra Y, Graterol-Rivas, Modesto E, \& Bautista-Sandoval, María J. (2017). Educación Superior y la Formación en Emprendimiento Interdisciplinario: Un Caso de Estudio. Formación Universitaria, 10(3), 11-20.

Echeverri, L., Valencia-Arias, Alejandro, BenjumeaArias, Martha, \& Toro, Andry Barrera-Del. (2018). Factores que inciden en la intención emprendedora del estudiantado universitario: Un análisis cualitativo. Revista Electrónica Educare, 22 (2), 160-178.

Fong, W., Curiel-Gómez, R., \& Brito-Carrillo, C. (2017). Aprendizaje significativo y su relación con la motivación intrínseca, escuela de procedencia y estrategias cognitivas en estudiantes de ingeniería. IPSA Scientia, Revista científica Multidisciplinaria, 2(1), 55-64.
Fong, W., Taron-Dunoyer, A., \& Zabaleta-Mesino, R. (2019). Nuevo liderazgo organizacional para fortalecer instituciones universitarias débilmente acopladas según Weick. IPSA Scientia, Revista científica Multidisciplinaria, 4(1), 60-70.

Geizzelez, M., \& Soto-Gómez, G. (2021). Creatividad, colaboración y confianza: Aptitud para la cultura innovativa en las redes de investigación estudiantil. IPSA Scientia, Revista científica Multidisciplinaria, 6(3), 33-43.

Linares, J., \& Geizzelez-Luzardo, M. (2016). Políticas científicas y tecnológicas para la gestión del conocimiento en instituciones universitarias. IPSA Scientia, Revista científica Multidisciplinaria, 1(1), 10-17.

Martínez, J., \& Garcés-Fuenmayor, J. (2021). Innovación en unidades universitarias de investigación: una mirada desde el desarrollo endógeno. IPSA Scientia, Revista científica Multidisciplinaria, 6(2), 26-34.

Molina, A., Rubio-Rodríguez GA, Bonilla Cortés V, Medina Bonilla J. (2020). Modelo de educación en emprendimiento. Un análisis desde la perspectiva de la comunidad educativa y empresarial. bol.redipe, 9(2), 145-62.

Paz, A., Pinto Aragón E, Mendoza Cataño C. (2015). Emprendimiento: Herramienta para la formación del emprendedor social en la Universidad de la Guajira. bol.redipe, 4(8), 79-85.

Rivera, R. (2019). "Particularidades del espíritu emprendedor en los jóvenes de la ciudad de Tarija". Revista Investigación y Negocios, 12(20), 115-158.

Salguero, N., García Salguero CP, Chiluisa Chiluisa MJ, Castro Bungacho SJ. (2017). Emprendimiento, universidad y sociedad. bol. redipe, 6(4), 218-25. 
Sánchez, J., Ward, Alexander, Hernández, Brizeida, \& Florez, Jenny Lizette. (2017). Educación emprendedora: Estado del arte. Propósitos y Representaciones, 5(2), 401-473.

Sandoval, M., Surdez Pérez, Edith Georgina, \& Pérez Sandoval, Abraham Gerardo. (2020). Nivel de la capacidad para el emprendimiento en estudiantes de ingeniería y arquitectura de una universidad pública del sureste de México. RIDE. Revista Iberoamericana para la Investigación y el Desarrollo Educativo, 11(21), e038.

Torres, L., \& Díaz-Ferrer, J. (2021). Inteligencias múltiples en el fortalecimiento del aprendizaje cooperativo efectivo. IPSA Scientia, Revista científica Multidisciplinaria, 6(1), 64-80.

Vega, J., Mera, C. (2016). Modelo de formación en emprendimiento social para Instituciones de Educación Superior en Colombia. Revista Escuela de Administración de Negocios, 81(2), 29-43.

Velasco, A. (2014) Reporte final presentado en el Seminario sobre aspectos cognitivos de la interacción verbal en el aula de clase. Revista Iberoamericana de Educación, 16(2), 17-28.

Vidal, J., Palencia Del Valle Z, Cardenas Fragozo NG. (2018). Elementos del emprendimiento social asociado al desarrollo endógeno de la población en condición de discapacidad ubicados en la zona de influencia: Maicao, Uribía y Manaure. bol.redipe, 7(6), 123-34. 